\title{
Double-blind evaluation of BACTEC and Buddemeyer-type radiorespirometric assays for in vitro screening of antileprosy agents
}

\author{
S G FRANZBLAU*, A N BISWAS, * P JENNER $†$ \& \\ M J COLSTON† \\ *Laboratory Research Branch, Gillis W. Long Hansen's Disease \\ Center, Carville, Louisiana 70721 USA and $\dagger$ National Institute for \\ Medical Research, The Ridgeway, Mill Hill, London NW7 1AAUK
}

\author{
Accepted for publication 31 January 1992
}

\begin{abstract}
Summary Two radiorespirometric assays, the BACTEC 460 and Buddemeyertype ${ }^{14} \mathrm{CO}_{2}$ detection systems, were evaluated in a double-blind manner for their ability to discriminate between authentic antileprosy agents and inactive compounds. Freshly harvested, nude-mouse derived Mycobacterium leprae were incubated in axenic media in the presence of coded test solutions prepared in a remote laboratory. Activity was assessed by comparing the rate of ${ }^{14} \mathrm{CO}_{2}$ evolution from $\left[1-{ }^{14} \mathrm{C}\right]$ palmitic acid to controls. Breaking the code revealed that both systems demonstrated a dose response to ethionamide, pefloxacin and rifampicin as well as sensitivity to dapsone. Most of the water, ethanol, sucrose, dabsyl chloride and riboflavin negative-control samples failed to effect a significant reduction in radiorespirometric activity. This study confirms the ability of the radiorespirometric assays to function as a primary drug screening system in leprosy.
\end{abstract}

\section{Introduction}

Historically, the screening of compounds for activity against Mycobacterium leprae has had to utilize the mouse footpad model ${ }^{1-3}$ because of the failure to cultivate the leprosy bacillus in vitro. The high cost, the length of time (6-12 months per experiment), the requirement for gram quantities of drug and the dependence on non-human pharmacokinetics ${ }^{4}$ have all motivated investigators to develop rapid in vitro systems which measure bacterial metabolism (following exposure to antimicrobial agents) as an index of viability. ${ }^{5-7}$

The ability of $M$. leprae to oxidize rapidly palmitic acid to carbon dioxide ${ }^{8-10}$ has been exploited by Franzblau et al. ${ }^{10-17}$ when screening for new antileprosy drugs, using two related assay systems: a Buddemeyer-type, liquid scintillation based, two-compartment system ${ }^{10}$ and an adaptation of the commercial BACTEC 460 system. ${ }^{11}$ These systems are very similar in that they both quantitate the rate of ${ }^{14} \mathrm{CO}_{2}$ evolution from ${ }^{14} \mathrm{C}$-labelled 
substrates. Both are 'automated' systems which do not require host cells, subsampling, extraction, filtration or other processing following the incubation of bacilli with drugs.

The present WHO-sponsored, double-blind study was undertaken to evaluate objectively the utility of these systems in screening for antileprosy drugs. Coded samples of compounds with known antileprosy activity, and control samples with no activity, were prepared in the London laboratory and sent to the Carville laboratory where they were tested in the two in vitro assays. In this way we were able to assess the reliability and reproducibility of the assays in screening for antileprosy activity.

\section{Methods}

The drug solutions were composed of stock solutions of rifampicin (RMP; $2.5 \mathrm{mg} / \mathrm{ml}$ in ethanol), ethionamide (ETH), dapsone (DDS; $5 \mathrm{mg} / \mathrm{ml}$ in ethanol), and pefloxacin (PEF; $5 \mathrm{mg} / \mathrm{ml}$ in water) and were prepared at NIMR (London). Triplicate $0.1 \mathrm{ml}$ aliquots of these stock solutions, together with $2 \cdot 5$-fold, 5-fold and 10-fold dilutions were provided for a subsequent 100 -fold dilution in the test media following filter sterilization. Negative control solutions consisted of distilled water, absolute ethanol, $1 \%$ sucrose, riboflavin $(0 \cdot 5$ $\mathrm{mg} / \mathrm{ml})$, and dabsyl chloride $(0 \cdot 1 \mathrm{mg} / \mathrm{ml})$. These last two acted as colour controls for ethionamide and rifampicin, respectively. The 70 test samples were coded, randomized, stored at $-20^{\circ} \mathrm{C}$ and shipped on dry ice to the Carville laboratory where they were held at $-20^{\circ} \mathrm{C}$ until used.

\section{LEPRAE INOCULUM}

$M$. leprae was harvested from the footpads of athymic nu/nu mice when the footpads had reached a bacillary load of approximately $10^{10} \mathrm{AFB}$. Footpads were surface decontaminated with iodine and ethanol, minced and homogenized in 7H12 medium (Middlebrook $7 \mathrm{H} 9$ broth, $0 \cdot 1 \%$ casitone (Difco), $1 \% \mathrm{w} / \mathrm{v}$ albumin and $5 \mu \mathrm{g} / \mathrm{ml}$ catalase). The bulk of tissue debris was removed by slow-speed centrifugation $\left(108 \times g, 5 \mathrm{~min}, 10^{\circ} \mathrm{C}\right)$ and the bacilli pelleted $\left(2710 \times g, 45 \mathrm{~min}, 10^{\circ} \mathrm{C}\right)$ and resuspended in $7 \mathrm{H} 12$ medium to approximately $10^{9} / \mathrm{ml}$. Cell counts were determined by the method of Shepard \& McRae. ${ }^{18}$ The suspensions were treated with $50 \mu \mathrm{g} / \mathrm{ml}$ ampicillin and $2.5 \mu \mathrm{g} / \mathrm{ml}$ amphotericin $\mathrm{B}$ for $4-5 \mathrm{~h}$ to eliminate contaminants. These agents have repeatedly been shown to be inactive against $M$. leprae. $^{10,19}$ Aliquots of the bacillary suspension were inoculated into Middlebrook 7H11 and Lowenstein-Jensen slants, tryptic soy and thioglycollate broths and blood agar to check for contaminants.

\section{BACTEC}

The $M$. leprae suspension was diluted to $10^{8} / \mathrm{ml}$ in $7 \mathrm{H} 12$ medium and $0 \cdot 1 \mathrm{ml}$ aliquots $\left(10^{7}\right.$ AFB) delivered via tuberculin syringe to BACTEC 12B media (4 ml in $20 \mathrm{ml}$ serum vials) supplemented with $50 \mu \mathrm{g} / \mathrm{ml}$ ampicillin and $2.5 \mu \mathrm{g} / \mathrm{ml}$ amphotericin B. The controls were 16 cultures that had received $0.1 \mathrm{ml} 7 \mathrm{H} 9$ broth. Other cultures received $0.1 \mathrm{ml}$ of coded test solutions in quadruplicate. All vials were flushed with $2.5 \%$ oxygen, $10 \%$ carbon dioxide, balance nitrogen using the BACTEC 460 instrument and were then incubated at $33^{\circ} \mathrm{C}$. The growth index ( $\mathrm{GI},{ }^{14} \mathrm{CO}_{2}$ evolution) was determined at weekly intervals for 3 weeks in 
the BACTEC 460 by flushing with the above gas mixture. Readings from week 2 were used in evaluating activity.

\section{BUDDEMEYER}

The $M$. leprae suspension was diluted to $10^{6} / \mathrm{ml}$ in $7 \mathrm{H} 12$ medium and $1 \mathrm{ml}$ aliquots $\left(10^{6}\right.$ AFB) dispensed to $6 \mathrm{ml} \mathrm{screw-capped} \mathrm{vials} \mathrm{(Wheaton,} \mathrm{Millville,} \mathrm{NJ,} \mathrm{USA).} \mathrm{The} \mathrm{controls}$ were 16 cultures who received $10 \mu 17 \mathrm{H} 9$ broth. Other cultures received $10 \mu \mathrm{l}$ of coded test solutions in quadruplicate. Vials, with loose caps, were then incubated for 2 weeks at $33^{\circ} \mathrm{C}$ under an atmosphere of $2.5 \%$ oxygen, $10 \%$ carbon dioxide in an incubator with automatic oxygen and carbon dioxide control capability, and 1 microCurie of $\left[1-{ }^{14} \mathrm{C}\right]$ palmitic acid ( $58 \mathrm{mCi} / \mathrm{mmole}$; New England Nuclear, Boston, MA, USA) was added to each vial in a volume of $10 \mu$ l ethanol. The glass vials, with loose caps, were then placed within wide-mouth scintillation vials (Poly-Q; Beckman, Brea, CA) containing a $2 \times 4 \mathrm{~cm}$ strip of Whatman No. 42 filter paper which had previously been dipped into a mixture of $20 \mathrm{ml}$ Liquifluor concentrate (New England Nuclear), $15 \mathrm{~g}$ PPO, $5 \mathrm{ml}$ Triton-X 100 and 5 $\mathrm{ml} 2 \mathrm{~N} \mathrm{NaOH}$ (in $\mathrm{MeOH}$ ). The entire assembly was incubated at $33^{\circ} \mathrm{C}$ and ${ }^{14} \mathrm{CO}_{2}$ evolution determined at daily intervals for 1 week by placing the double vial assemblies in a liquid scintillation counter. Cumulative counts per minute from the day 7 reading were used in evaluating activity.

\section{STATISTICAL EVALUATION}

Statistical significance was determined by the Student's $t$-test.

\section{Results}

BACTEC

Using a $p$ value of 0.05 as a cut-off for active substances, there were 6 false positives out of 22 non-antileprosy substances (Table 1). While 11 of 12 DDS samples were active, there was little, if any, dose-response over the entire range of $0 \cdot 1-5 \cdot 0 \mu \mathrm{g} / \mathrm{ml}$. Both ETH and PEF displayed no activity at $0 \cdot 1 \mu \mathrm{g} / \mathrm{ml} ; 2$ of 3 samples were active at $0.5 \mu \mathrm{g} / \mathrm{ml}$ and all 2.0 and $5.0 \mu \mathrm{g} / \mathrm{ml}$ samples effected significant reductions compared to controls. The dose response was more apparent with PEF than with ETH. All RMP concentrations demonstrated highly significant activity and effected a clear dose-response.

\section{BUDDEMEYER}

Only 1 of the 22 non-antileprosy substances appeared active, possibly due to the large SD obtained with this sample (A20) (Table 2). Results with DDS were variable, although 2 of 3 samples were positive at $0 \cdot 5,2 \cdot 0$ and $5 \cdot 0 \mu \mathrm{g} / \mathrm{ml}$. Both ETH and PEF gave inconclusive results at $0 \cdot 1 \mu \mathrm{g} / \mathrm{ml}$ and significant dose-responsive activity at the 3 higher concentrations. All concentrations of RMP again demonstrated highly significant, dose-dependent activity. 
Table 1. BACTEC system

\begin{tabular}{|c|c|c|c|c|c|}
\hline $\begin{array}{l}\text { Coded } \\
\text { sample }\end{array}$ & $\begin{array}{l}\text { De-coded sample } \\
\text { and concentration } \\
(\mu \mathrm{g} / \mathrm{ml})\end{array}$ & Mean & $\mathrm{SD}$ & $p$ & Rating \\
\hline Control & & 746 & 113 & & \\
\hline Heat killed & & 2 & 1 & $<0.001$ & \\
\hline A 13 & Water & 670 & 39 & $0 \cdot 212$ & Inactive \\
\hline A 18 & Water & 684 & 63 & $0 \cdot 315$ & Inactive \\
\hline A 22 & Water & 574 & 108 & 0.014 & Active*† \\
\hline A 25 & Water & 613 & 48 & 0.036 & Active* + \\
\hline A 32 & Water & 643 & 37 & 0.094 & Inactive \\
\hline A65 & Water & 576 & 27 & 0.010 & Active*† \\
\hline A 10 & Sucrose & 639 & 50 & 0.088 & Inactive \\
\hline A 37 & Sucrose & 654 & 35 & $0 \cdot 192$ & Inactive \\
\hline A08 & Ethanol & 633 & 31 & 0.068 & Inactive \\
\hline A 12 & Ethanol & 732 & 44 & $0 \cdot 808$ & Inactive \\
\hline A 23 & Ethanol & 626 & 49 & 0.057 & Inactive \\
\hline A 36 & Ethanol & 648 & 45 & $0 \cdot 113$ & Inactive \\
\hline A 39 & Ethanol & 642 & 25 & $0 \cdot 100$ & Inactive \\
\hline A59 & Ethanol & 752 & 51 & 0.926 & Inactive \\
\hline A04 & Dabsyl Cl & 725 & 9 & 0.724 & Inactive \\
\hline A52 & Dabsyl Cl & 807 & 32 & $0 \cdot 308$ & Inactive \\
\hline A61 & Dabsyl Cl & 736 & 38 & $0 \cdot 864$ & Inactive \\
\hline A69 & Dabsyl Cl & 614 & 21 & 0.035 & Active* + \\
\hline A 20 & Riboflavin & 698 & 77 & 0.437 & Inactive* \\
\hline A26 & Riboflavin & 591 & 37 & 0.017 & Active*† \\
\hline A 38 & Riboflavin & 703 & 117 & 0.513 & Inactive \\
\hline A70 & Riboflavin & 528 & 76 & 0.002 & Active* $\dagger$ \\
\hline $\mathrm{A} 03$ & DDS $0 \cdot 1$ & 564 & 12 & 0.006 & Active \\
\hline A34 & DDS $0 \cdot 1$ & 516 & 12 & 0.001 & Active* \\
\hline A 57 & DDS $0 \cdot 1$ & 611 & 18 & 0.032 & Active* \\
\hline A02 & DDS 0.5 & 501 & 48 & 0.001 & Active \\
\hline A 48 & DDS 0.5 & 669 & 41 & $0 \cdot 204$ & Inactive* \\
\hline A56 & DDS 0.5 & 599 & 3 & 0.021 & Active \\
\hline A01 & $\operatorname{DDS} 2 \cdot 0$ & 512 & 26 & 0.001 & Active \\
\hline A 31 & DDS $2 \cdot 0$ & 462 & 24 & $<0.001$ & Active* \\
\hline A67 & $\operatorname{DDS} 2 \cdot 0$ & 480 & 15 & $<0.001$ & Active \\
\hline A 27 & DDS $5 \cdot 0$ & 476 & 13 & $<0.001$ & Active \\
\hline A 50 & DDS $5 \cdot 0$ & 588 & 2 & 0.014 & Active* \\
\hline A68 & DDS 5.0 & 425 & 32 & $<0.001$ & Active \\
\hline A 16 & ЕTH $0 \cdot 1$ & 651 & 43 & $0 \cdot 123$ & Inactive* \\
\hline A 44 & ETH $0 \cdot 1$ & 775 & 47 & 0.630 & Inactive \\
\hline A 55 & ETH $0 \cdot 1$ & 759 & 19 & $0 \cdot 824$ & Inactive \\
\hline A 43 & ETH 0.5 & 635 & 165 & $0 \cdot 129$ & Inactive* \\
\hline A62 & ETH 0.5 & 570 & 22 & 0.007 & Active \\
\hline A64 & ETH 0.5 & 570 & 17 & 0.007 & Active \\
\hline A07 & ETH $2 \cdot 0$ & 537 & 46 & 0.002 & Active \\
\hline A40 & ETH $2 \cdot 0$ & 469 & 56 & $<0.001$ & Active \\
\hline A41 & ETH $2 \cdot 0$ & 551 & 70 & 0.005 & Active \\
\hline Al4 & ETH 5.0 & 449 & 36 & $<0.001$ & Active \\
\hline A 30 & ETH 5.0 & 395 & 50 & $<0.001$ & Active \\
\hline A 47 & ETH $5 \cdot 0$ & 503 & 43 & 0.001 & Active \\
\hline A19 & PEF $0 \cdot 1$ & 728 & 48 & 0.758 & Inactive* \\
\hline A 42 & PEF $0 \cdot 1$ & 816 & 49 & $0 \cdot 316$ & Inactive \\
\hline A49 & PEF $0 \cdot 1$ & ND & & & \\
\hline
\end{tabular}


Table 1. (Contd.)

\begin{tabular}{|c|c|c|c|c|c|}
\hline $\begin{array}{l}\text { Coded } \\
\text { sample }\end{array}$ & $\begin{array}{l}\text { De-coded sample } \\
\text { and concentration } \\
\qquad(\mu \mathrm{g} / \mathrm{ml})\end{array}$ & Mean & SD & $p$ & Rating \\
\hline All & PEF 0.5 & 643 & 61 & $0 \cdot 100$ & Inactive* \\
\hline A 15 & PEF 0.5 & 616 & 79 & 0.047 & Active \\
\hline A29 & PEF 0.5 & 604 & 22 & $0 \cdot 025$ & Active \\
\hline A 17 & PEF $2 \cdot 0$ & 536 & 50 & 0.002 & Active \\
\hline A 28 & PEF $2 \cdot 0$ & 457 & 35 & $<0.001$ & Active \\
\hline A 45 & PEF $2 \cdot 0$ & 561 & 64 & 0.006 & Active \\
\hline A09 & PEF $5 \cdot 0$ & 362 & 29 & $<0.001$ & Active \\
\hline A 53 & PEF $5 \cdot 0$ & 418 & 5 & $<0.001$ & Active \\
\hline A66 & PEF 5.0 & 318 & 46 & $<0.001$ & Active \\
\hline A 24 & RMP 0.05 & 458 & 32 & $<0.001$ & Active \\
\hline A 33 & RMP 0.05 & 440 & 41 & $<0.001$ & Active \\
\hline A63 & RMP 0.05 & ND & & & Active \\
\hline A06 & RMP 0.25 & 394 & 26 & $<0.001$ & Active \\
\hline A 35 & RMP $0 \cdot 25$ & 290 & 37 & $<0.001$ & Active \\
\hline A54 & RMP 0.25 & 395 & 28 & $<0.001$ & Active \\
\hline A 21 & RMP $1 \cdot 0$ & 204 & 35 & $<0.001$ & Active \\
\hline A 58 & RMP 1.0 & 262 & 27 & $<0.001$ & Active \\
\hline A60 & RMP $1 \cdot 0$ & 258 & 14 & $<0.001$ & Active \\
\hline A05 & RMP $2 \cdot 5$ & 226 & 10 & $<0.001$ & Active \\
\hline A46 & RMP $2 \cdot 5$ & 222 & 37 & $<0.001$ & Active \\
\hline A 51 & RMP $2 \cdot 5$ & 212 & 8 & $<0.001$ & Active \\
\hline
\end{tabular}

For controls, $n=16$; for test samples, $n=4$.

Rating criteria: active $=p<0.05$; inactive $=p>0.05 \mathrm{vs}$. controls.

*, Apparent disagreement with results of Buddemeyer assay.

ND, not determined; $\uparrow$, false positive result.

\section{Discussion}

This double-blind, 2-laboratory evaluation of these in vitro radiorespirometric systems confirms previous reports on the ability of these assays to detect activity of established antileprosy drugs at concentrations below peak obtainable plasma levels. ${ }^{10-12}$ All of the known antileprosy compounds were found to be active at the concentrations which they would be predicted to be active and there was good concordance between replicate samples. Results from earlier studies and this report suggest a correlation between degree of drug activity in the mouse footpad ${ }^{19-24}$ and radiorespirometric systems. ${ }^{11,12,14,16}$ In general, highly active, potent drugs such as RMP produced lower levels of ${ }^{14} \mathrm{CO}_{2}$ than bacteriostatic agents such as DDS. Since all concentrations of RMP were active in both Buddemeyer and the BACTEC systems, we were unable to determine an MIC. The MIC of ethionamide, as measured by the in vitro systems, appeared to be between $0 \cdot 1$ and 0.5 $\mu \mathrm{g} / \mathrm{ml}$ (compared to $0.05 \mu \mathrm{g} / \mathrm{ml}$ as estimated by the mouse footpad system). All concentrations of DDS were active in the BACTEC system, whereas in the Buddemeyer system occasional samples at all concentrations were found to be inactive, making it difficult to compare the MIC in vitro with that estimated using the mouse footpad technique (approximately $0.003 \mu \mathrm{g} / \mathrm{ml}$ ). Pefloxacin appears to have an MIC of approximately $0.5 \mu \mathrm{g} / \mathrm{ml}$ in vitro. 
Table 2. Buddemeyer system

\begin{tabular}{|c|c|c|c|c|c|}
\hline $\begin{array}{l}\text { Coded } \\
\text { sample }\end{array}$ & $\begin{array}{l}\text { De-coded sample } \\
\text { and concentration } \\
\qquad(\mu \mathrm{g} / \mathrm{ml})\end{array}$ & Mean & SD & $p$ & Rating \\
\hline Control & & 7213 & 992 & & \\
\hline Heat killed & & 148 & 10 & $<0.001$ & \\
\hline A 13 & Water & 6704 & 331 & 0.333 & Inactive \\
\hline A 18 & Water & 6671 & 552 & $0 \cdot 313$ & Inactive \\
\hline A 22 & Water & 6731 & 982 & $0 \cdot 395$ & Inactive* \\
\hline A 25 & Water & 6833 & 243 & $0 \cdot 465$ & Inactive* \\
\hline A 32 & Water & 7325 & 958 & 0.842 & Inactive \\
\hline A65 & Water & 6963 & 987 & 0.656 & Inactive* \\
\hline A 10 & Sucrose & 7257 & 605 & 0.935 & Inactive \\
\hline A 37 & Sucrose & 6501 & 533 & $0 \cdot 188$ & Inactive \\
\hline A08 & Ethanol & 6693 & 758 & $0 \cdot 343$ & Inactive \\
\hline A 12 & Ethanol & 7384 & 435 & 0.745 & Inactive \\
\hline A 23 & Ethanol & 6361 & 603 & $0 \cdot 174$ & Inactive \\
\hline A 36 & Ethanol & 7316 & 1300 & 0.876 & Inactive \\
\hline A 39 & Ethanol & 7444 & 1251 & 0.696 & Inactive \\
\hline A59 & Ethanol & 7879 & 306 & $0 \cdot 210$ & Inactive \\
\hline A04 & Dabsyl Cl & 6272 & 1496 & $0 \cdot 141$ & Inactive \\
\hline A52 & Dabsyl Cl & 6548 & 466 & $0 \cdot 215$ & Inactive \\
\hline A61 & Dabsyl Cl & 6896 & 1003 & 0.575 & Inactive \\
\hline A69 & Dabsyl Cl & 8143 & 1165 & $0 \cdot 122$ & Inactive* \\
\hline A 20 & Riboflavin & 5399 & 1971 & 0.023 & Active*† \\
\hline A 26 & Riboflavin & 7092 & 268 & $0 \cdot 814$ & Inactive* \\
\hline A 38 & Riboflavin & 6760 & 681 & 0.403 & Inactive \\
\hline A70 & Riboflavin & 7252 & 544 & 0.941 & Inactive* \\
\hline A03 & DDS $0 \cdot 1$ & 5623 & 648 & 0.007 & Active \\
\hline A 34 & DDS $0 \cdot 1$ & 6258 & 330 & 0.078 & Inactive* \\
\hline A 57 & DDS $0 \cdot 1$ & 6310 & 213 & 0.093 & Inactive* \\
\hline A02 & DDS 0.5 & 5543 & 100 & 0.004 & Active \\
\hline A 48 & DDS 0.5 & 5392 & 804 & 0.003 & Active* \\
\hline A56 & DDS 0.5 & 5746 & 781 & 0.014 & Active \\
\hline A01 & DDS $2 \cdot 0$ & 5439 & 828 & 0.004 & Active \\
\hline A 31 & $\operatorname{DDS} 2 \cdot 0$ & 6621 & 414 & $0 \cdot 265$ & Inactive* \\
\hline A67 & DDS $2 \cdot 0$ & 6080 & 814 & $0 \cdot 050$ & Active \\
\hline A 27 & DDS $5 \cdot 0$ & 5805 & 1204 & 0.025 & Active \\
\hline A 50 & DDS $5 \cdot 0$ & 6268 & 1076 & $0 \cdot 110$ & Inactive* \\
\hline A68 & DDS 5.0 & 5193 & 290 & 0.001 & Active \\
\hline A 16 & ЕТН $0 \cdot 1$ & 5884 & 1235 & 0.034 & Active* \\
\hline A 44 & ETH $0 \cdot 1$ & 6501 & 335 & $0 \cdot 181$ & Inactive \\
\hline A55 & ETH $0 \cdot 1$ & 7035 & 854 & 0.746 & Inactive \\
\hline A43 & ETH 0.5 & 5038 & 1131 & 0.001 & Active* \\
\hline A 62 & ETH 0.5 & 5791 & 456 & 0.013 & Active \\
\hline A64 & ETH 0.5 & 5296 & 1626 & 0.007 & Active \\
\hline A07 & ETH $2 \cdot 0$ & 1699 & 460 & $<0.001$ & Active \\
\hline A 40 & ETH $2 \cdot 0$ & 1953 & 570 & $<0.001$ & Active \\
\hline A41 & ETH $2 \cdot 0$ & ND & & & Active \\
\hline A 14 & ETH 5.0 & 1086 & 39 & $<0.001$ & Active \\
\hline A 30 & ETH 5.0 & 1163 & 237 & $<0.001$ & Active \\
\hline A 47 & ETH 5.0 & 1270 & 85 & $<0.001$ & Active \\
\hline A19 & PEF $0 \cdot 1$ & 5789 & 1252 & 0.025 & Active* \\
\hline A42 & PEF $0 \cdot 1$ & ND & & & \\
\hline A49 & PEF $0 \cdot 1$ & 8480 & 550 & 0.026 & Inactive \\
\hline
\end{tabular}


Table 2. (Contd.)

\begin{tabular}{|c|c|c|c|c|c|}
\hline $\begin{array}{l}\text { Coded } \\
\text { sample }\end{array}$ & $\begin{array}{l}\text { De-coded sample } \\
\text { and concentration } \\
(\mu \mathrm{g} / \mathrm{ml})\end{array}$ & Mean & SD & $p$ & Rating \\
\hline A 11 & PEF 0.5 & 5206 & 644 & 0.001 & Active* \\
\hline A 15 & PEF 0.5 & 4732 & 469 & $<0.001$ & Active \\
\hline A 29 & PEF 0.5 & 5527 & 961 & 0.007 & Active \\
\hline A 17 & PEF 2.0 & 3319 & 430 & $<0.001$ & Active \\
\hline A 28 & PEF $2 \cdot 0$ & 3438 & 264 & $<0.001$ & Active \\
\hline A 45 & PEF 2.0 & 3136 & 717 & $<0.001$ & Active \\
\hline A09 & PEF 5.0 & 2506 & 187 & $<0.001$ & Active \\
\hline A53 & PEF 5.0 & 2458 & 19 & $<0.001$ & Active \\
\hline A66 & PEF 5.0 & 2608 & 307 & $<0.001$ & Active \\
\hline A 24 & RMP 0.05 & 4179 & 557 & $<0.001$ & Active \\
\hline A 33 & RMP 0.05 & 4423 & 922 & $<0.001$ & Active \\
\hline A63 & RMP 0.05 & 5346 & 389 & 0.002 & Active \\
\hline A06 & RMP 0.25 & 2174 & 388 & $<0.001$ & Active \\
\hline A 35 & RMP 0.25 & 2266 & 348 & $<0.001$ & Active \\
\hline A54 & RMP 0.25 & 2082 & 57 & $<0.001$ & Active \\
\hline A 21 & RMP $1 \cdot 0$ & 453 & 139 & $<0.001$ & Active \\
\hline A58 & RMP $1 \cdot 0$ & 832 & 122 & $<0.001$ & Active \\
\hline A 60 & RMP $1 \cdot 0$ & 802 & 168 & $<0.001$ & Active \\
\hline A05 & RMP 2.5 & 633 & 77 & $<0.001$ & Active \\
\hline A 46 & RMP 2.5 & 696 & 47 & $<0.001$ & Active \\
\hline A 51 & RMP $2 \cdot 5$ & 631 & 87 & $<0.001$ & Active \\
\hline
\end{tabular}

For controls, $n=16$; for test samples, $n=4$.

Rating criteria: active $=p<0.05$; inactive $=p>0.05$ vs. controls.

*, Apparent disagreement with results of BACTEC assay.

ND, not determined; $†$ false positive result.

Up to now the radiorespirometric systems evaluated in this report have been used to screen 6 macrolides, ${ }^{14} 17$ clofazimine derivatives, ${ }^{15,17} 20$ fluoroquinolones, ${ }^{16} 1$ tetracycline, ${ }^{11} 5$ aminoglycosides, 3 rifamycins and 2 lincosamides ${ }^{12}$ for activity against $M$. leprae. Thus, 2 agents, clarithromycin ${ }^{14}$ and sparfloxacin, ${ }^{16}$ were first recognized as being the most active clinically relevant agents in their respective classes and are currently in clinical trials in leprosy. Another potential antileprotic identified by radiorespirometry is fusidic acid, ${ }^{13}$ an established antibiotic which has poor pharmacokinetics in mice (in contrast to humans) and is inactive against $M$. leprae in the mouse footpad model (Colston, unpublished results).

There was fairly good agreement (79\%) between the BACTEC and Buddemeyer systems, especially considering that the two assays were evaluated in separate experiments at different times using different nude mouse-derived inocula. The BACTEC system requires approximately 10 times more bacilli than the Buddemeyer-type system to obtain usable readings. This relatively lower sensitivity also results in smaller differences observed between control and drug-treated samples than that observed in the Buddemeyer system. The BACTEC system, however, appears to be more sensitive to weaklyactive agents such as DDS and results in lower variance among replicate samples. This may be due to the ability to produce efficiently and maintain the desired (microaerophilic) environment using the BACTEC 460 instrument, which appears optimal for maintenance of $M$. leprae viability in vitro. ${ }^{25} \mathrm{With}$ either test (consisting of approximately 300 vials -70 
samples in quadruplicate plus 4 heat-killed and 16 drug-free controls), the numbers evaluated in the present study can be read within a normal 8 hour working day.

\section{Conclusion}

Radiorespirometry, using either the BACTEC 460 or Buddemeyer ${ }^{14} \mathrm{CO}_{2}$ detection systems, is capable of differentiating between antileprosy agents and inactive substances following a 2-week incubation of freshly harvested, viable leprosy bacilli under appropriate incubation conditions.

\section{Acknowledgments}

This study was partially supported by the UNDP/World Bank/WHO Special Programme for Research and Training in Tropical Diseases; Project No. 890460.

\section{References}

1 Colston MJ, Hilson GRF, Banerjee DK. The 'proportional bactericidal test': a method for assessing bactericidal activity of drugs against Mycobacterium leprae in mice. Lepr Rev, 1978; 49: 7-15.

2 Shepard CC. A kinetic method for the study of activity of drugs against Mycobacterium leprae in mice. Int $J$ Lepr, 1967; 35: 429-35.

3 Shepard CC, Chang YT. Effect of several anti-leprosy drugs on multiplication of human leprosy bacilli in footpads of mice. Proc Soc Exp Biol Med, 1962; 109: 636-38.

${ }^{4}$ Shepard CC, van Landingham RM, Walker LL. Recent studies of antileprosy drugs. Lepr Rev, 1983; 54: 23S-30S.

5 Barclay R, Wheeler PR. Metabolism of mycobacteria in tissues. In: The biology of the mycobacteria. Ratledge C, Stanford J, Grange F. (eds), London: Academic Press, 1989, Vol. 3, pp. 37-106.

${ }^{6}$ Hastings RC, Franzblau SG. Chemotherapy of leprosy. Annu Rev Pharmacol Toxicol, 1988; 28: 231-45.

7 Hastings RC, Gillis TP, Krahenbuhl JL, Franzblau SG. Leprosy. Clin Microbiol Rev, 1988; 1: 330-48.

8 Ishaque M. Direct evidence for the oxidation of palmitic acid by host-grown Mycobacterium leprae. Res Microbiol, 1989; 140: 83-93.

9 Wheeler PR, Ratledge C. Use of carbon sources for lipid biosynthesis in Mycobacterium leprae: a comparison with other pathogenic mycobacteria. J Gen Microbiol, 1988; 134: 2111-21.

${ }^{10}$ Franzblau SG. Oxidation of palmitic acid by Mycobacterium leprae in an axenic medium. J Clin Microbiol, 1988; 26: 18-21.

11 Franzblau SG. Drug susceptibility testing of Mycobacterium leprae in the BACTEC 460 system. Antimicrob Agents Chemother, 1989; 33: 2115-17.

12 Franzblau SG. In vitro activities of aminoglycosides, linocsamides and rifamycins against Mycobacterium leprae. Antimicrob Agents Chemother, 1991; 35: 1232-34.

13 Franzblau SG, Biswas AN. Fusidic acid is highly active against extracellular and intracellular $M$ ycobacterium leprae. Antimicrob Agents Chemother, 1992; 36: 92-94.

14 Franzblau SG, Hastings RC. In vitro and in vivo activities of macrolides against Mycobacterium leprae in vitro. Antimicrob Agents Chemother, 1988; 32: 1583-85.

15 Franzblau SG, O’Sullivan JF. Structure-activity relationships of selected phenazines against Mycobacterium leprae in vitro. Antimicrob Agents Chemother, 1988; 32: 1583-85.

16 Franzblau SG, White KE. Comparative in vitro activity of 20 fluoroquinolones against Mycobacterium leprae. Antimicrob Agents Chemother, 1990; 34: 229-31.

17 Franzblau SG, White KE, O'Sullivan JF. Structure-activity relationships of tetramethylpiperidinesubstituted phenazines against Mycobacterium leprae in vitro. Antimicrob Agents Chemother, 1989; 33: 2004-5.

18 Shepard CC, McRae DH. A method for counting acid-fast bacteria. Int J Lepr, 1968; 36: 78-82.

19 Shepard CC. A survey of the drugs with activity against M. leprae in mice. Int J Lepr, 1971; 39: 340-48. 
20 Colston MJ, Ellard GA, Gammon PT. Drugs for combined therapy: experimental studies on the antileprosy activity of ethionamide and prothionamide, and a general review. Le pr Rev, 1978; 499: 115-26.

${ }^{21}$ Gelber RH, Siu P, Tsang M, Murray LP. Activities of various macrolide antibiotics against Mycobacterium leprae in mice. Antimicrob Agents Chemother, 1991; 35: 760-3.

22 Hastings RC, Jacobson RR. Activity of ansamycin against Mycobacterium leprae in mice. Lancet, 1983; ii: 1079-80.

23 Ji B, Perani EG, Grosset JH. Effectiveness of clarithromycin and minocycline alone and in combination against experimental Mycobacterium leprae infection in mice. Antimicrob Agents Chemother, 1991; 35: $579-81$.

24 Tsutsumi S, Gidoh M. Studies on the development of novel antileprous chemotherapeutics using nude mice with special reference to a new quinolone carboxylic acid, AT-4140. Jap J Le pr, 1989; 58: 250-58.

${ }^{25}$ Franzblau SG, Harris EB. Biophysical optima for metabolic maintenance in Mycobacterium leprae. J Clin Microbiol, 1988; 26: 1124-29.

Lepr Rev (1992) 63, 125-133

\title{
Évaluation en double-aveugle des tests radiorespirométriques BACTEC et type Buddemeyer pour le triage in vitro des agents antilépreux
}

\author{
S G Franzblau, A N Biswas, P Jenner eì M J Colston
}

Résumé Nous avons comparé deux essais radiorespirométriques, le système BACTEC 460 et le système type Buddemeyer de détection de ${ }^{14} \mathrm{CO}_{2}$, en double-aveugle, pour évaluer leur aptitude à différencier entre les agents antilépreux authentiques et les composés inactifs. Mycobacterium leprae provenant de souris "nude", fraîchement récolté, a été incubé en milieu axénique en présence des solutions à tester identifiées par code et préparées dans un laboratoire éloigné. L'activité a été déterminée en comparant le contôle du taux d'évolution de ${ }^{14} \mathrm{CO}_{2}$ partir de l'acide $\left(1-{ }^{14} \mathrm{C}\right)$ palmitique. En décodant, nous avons observé que les deux systèmes présentaient une résponse proportionnée à la dose à l'éthionamide, la pefloxacine et la rifampicine, de même qu'une sensibilité à la dapsone. La plupart des échantillons témoins-négatifs eau, éthanol, sucrose, chlorure de dabsyl et riboflavine n'ont produit aucune réduction significative de l'activité radiorespirométrique. Cette étude confirme l'aptitude des essais radiorespirométriques à fonctionner comme un système de triage primaire des agents antilépreux.

\section{La evaluación doble-ciego de las pruebas radiorespirométricos BACTEC y de tipo Buddemeyer en los estudios in vitro de los agentes contra la lepra}

\author{
S G Franzblau, A N Biswas, P Jenner y M J Colston
}

Resumen Dos pruebas radiorespirométricas, los sistemas de detección de ${ }^{14} \mathrm{CO}_{2}$ BACTEC 460 y el tipo Buddemeyer, fueron evaluados de un modo doble-ciego para su habilidad de discriminar entre agentes verdaderamente antileprosos y los compuestos inactivos. Mycobacterium leprá recién obtenido de ratones desnudos fue incubado in medios axénicos, en la presencia de soluciones de prueba codificadas, preparadas en un laboratorio remoto. Se evaluó la actividad comparando la velocidad de evolución de ${ }^{14} \mathrm{CO}_{2}$ del ácido $\left[1-{ }^{14} \mathrm{C}\right]$ palmítico. Después de revelar el código, ambos sistemas mostraron una respuesta de dósis a la etionamida, pefloxacina y rifampicina, además de sensibilidad a la dapsona. La mayoría de muestras de control negativo, de agua, etanol, sucrosa, cloruro de dabsilo y riboflavina no lograron una reducción significativa de actividad radiorespirométrica. El estudio confirma la manera en que las pruebas radiorespirométricas pueden funcionar como un sistema de examen primario para drogas contra la lepra. 